\title{
FACTORES ASOCIADOS AL NIVEL DE CONOCIMIENTO SOBRE LACTANCIA MATERNA EXCLUSIVA EN MADRES PRIMÍPARAS ATENDIDAS POR TELECONSULTA - INSTITUTO NACIONAL MATERNO PERINATAL EN EL AÑO 2020
}

\section{FACTORS ASSOCIATED WITH THE LEVEL OF KNOWLEDGE ABOUT EXCLUSIVE BREASTFEEDING IN FIRST MOTHERS SERVED BY TELECONSULTATION - NATIONAL MATERNAL PERINATAL INSTITUTE IN 2020}

\author{
Pablo Vizquerra-Guevara1,a Juan Carlos Ezequiel Roque Quezada ${ }^{1,2, b}$ Israel Armando Guerra Cuyutupac ${ }^{3}$ \\ Claudia Veralucia Saldaña Díaz ${ }^{2,4}$
}

\begin{abstract}
RESUMEN
Objetivo: Determinar el nivel de conocimiento sobre lactancia materna exclusiva en madres primíparas atendidas por teleconsulta del Instituto Nacional Materno Perinatal en año 2020 y sus factores asociados. Materiales y métodos: Estudio de enfoque cuantitativo, diseño descriptivo, observacional, transversal, prospectivo y analítico. La población se compuso de un total de 1000 madres primíparas atendidas por teleconsulta en el Instituto Nacional Materno Perinatal, tomando una muestra representativa de 240 participantes. Se empleó un instrumento confiable y valido para medir el conocimiento en lactancia materna exclusiva, se realizaron pruebas estadísticas de asociación y un modelo de regresión de Poisson bivariado y múltiple para obtener la razón de prevalencias crudas y ajustadas y sus intervalos de confianza al 95\% para los factores asociados y las categorías de lactancia materna exclusiva. Resultados: se encontró un $75.7 \%$ de nivel de conocimiento sobre lactancia materna exclusiva alto, con IC $95 \%$ de $69.5-81.1 \%$. Se encontró una asociación estadísticamente significativa para las variables gestante añosa (RPa 1.21 IC95\% 1.05 - 1.39) y grado de instrucción superior (RPa 1.22 IC 95\% 1.02 - 1.44) Conclusiones: un nivel de instrucción alto y la gestación añosa se identificaron como factores asociados a un nivel de conocimiento alto sobre lactancia materna exclusiva en madre primíparas atendidas por teleconsulta del Instituto Nacional Materno Perinatal en año 2020.
\end{abstract}

Palabras clave: Lactancia materna; Leche humana; Lactancia; Relaciones madre-hijo; Conocimiento; Teleconsulta (Fuente: DeCS BIREME).

\begin{abstract}
Objective: To determine the factors associated with the level of knowledge about exclusive breastfeeding in first-time mothers attended by teleconsultation of the National Maternal-Perinatal Institute in 2020. Materials and methods: Study with a quantitative approach, descriptive, observational, cross-sectional, and analytical design. The population was made up of a total of 1000 primiparous mothers attended by teleconsultation at the National Maternal Perinatal Institute, taking a representative sample of 240. A reliable and valid instrument was used to measure knowledge in exclusive breastfeeding, statistical association tests were performed and bivariate and multiple Poisson regression model to obtain the raw and adjusted prevalence ratio and their 95\% confidence intervals for the associated factors and exclusive breastfeeding categories. Results: a high $75.7 \%$ level of knowledge about exclusive breastfeeding was found, with a $95 \% \mathrm{Cl}$ of $69.5 \%$ $81.1 \%$. A statistically significant association was found for the variables of the elderly pregnant woman (PRa $1.2195 \% \mathrm{Cl}$ 1.05 - 1.39) and higher education level (PRa $1.2295 \% \mathrm{Cl} 1.02-1.44)$. Conclusions: a high level of education and elderly pregnancy were identified as associated factors at a high level of knowledge about exclusive breastfeeding in primiparous mothers attended by teleconsultation from the National Maternal-Perinatal Institute in 2020
\end{abstract}

Keywords: Breast Feeding, Human milk, lactation, mother-child relations, knowledge, Teleconsultation (Source: MeSH NLM).

\section{INTRODUCCIÓN}

El presente artículo aborda la problemática del déficit de nutrición y alimentación incorrecta que se le da a los recién nacidos, centrándose en la lactancia materna.
Para todo recién nacido la leche materna es el alimento fundamental para su nutrición ${ }^{1}$. Las mujeres que han dado de lactar exclusivamente tienen hijos más saludables y padecen de menos enfermedades². Además existe una importancia en la relación madre-hijo durante la lactancia,

\footnotetext{
Facultad de Medicina Humana, Universidad Ricardo Palma. Lima, Perú

Escuela profesional de Medicina Humana-Universidad Privada San Juan Bautista, Filial Chincha

Universidad Científica del Sur, Facultad de Medicina; Departamento de Anatomía Humana, Lima-Perú

Instituto Nacional Materno Perinatal

Médico Investigadora

Magister en medicina

D Código ORCID: https://orcid.org/0000-0002-1886-0426, Juan Carlos Ezequiel Roque Quezada

Citar como: Citar como: Vizquerra-Guevara P, Roque Quezada JCE, Guerra Cuyutupac IA, Saldaña Díaz CV. Factores asociados al nivel de conocimiento sobre lactancia materna exclusiva en madres primíparas atendidas por teleconsulta - Instituto Nacional Materno Perinatal en el año 2020. Rev Peru Investig Matern Perinat. 2021;10(1): 14-18

DOI https://doi.org/10.33421/inmp.2021222
}

Recibido: 10-01-2021 Aprobado: 31-03-2021 
ya que es fundamental para un correcto desarrollo en el ámbito afectivo y social ${ }^{3}$.

La lactancia materna debe realizarse exclusivamente hasta los 6 primeros meses ${ }^{4}$, después se debe de continuar con la alimentación complementaria para asegurar los nutrientes que están ausentes en la leche materna ${ }^{5}$. Cabe resaltar que la lactancia materna no solo trae beneficios para el lactante sino también para la madre que van más allá de la satisfacción emocional ${ }^{6}$, como la reducción de la hemorragia post parto y la disminución del riesgo de sufrir cáncer de mama y ovario ${ }^{7}$.

En el Perú hay una alta prevalencia de abandono de lactancia materna, que está relacionada con la creencia errada sobre este tema ${ }^{8}$, a pesar de los esfuerzos realizados por los establecimientos de salud por difundir este tema, no se está logrando el objetivo de que las madres manejen y sepan adecuadamente todo lo referente a los beneficios que trae la lactancia materna. Con esto se evidencia que falta reforzar el tema de asesoramiento sobre lactancia materna exclusiva por parte de los centros de salud ${ }^{9}$.

Esto nos llevó a estudiar los factores asociados al nivel de conocimiento sobre lactancia materna exclusiva en madres primíparas atendidas por teleconsulta del Instituto Nacional Materno Perinatal en el año 2020 con la finalidad de dar a conocer al sistema de salud la necesidad de poner énfasis promover la lactancia materna exclusiva y conocer lo factores que se asocian a esta, este es un tema trascendente que beneficiara a toda la sociedad, ya que los beneficios son el ahorro en formulas maternizadas, la sustentabilidad medio ambiental al no usar biberones, el impacto económico del aumento de la inteligencia por lactancia materna, entre otros ${ }^{10}$.

Con el presente estudio se observa la importancia de llevar a cabo acciones de promoción de lactancia materna mediante la transferencia de información adecuada y abordaje de problemas y factores habituales que ponen en riesgo la lactancia, esto mediante la formación de grupos de apoyo con la finalidad de mejorar las prácticas y poder prevenir posibles problemas importantes durante esta etapa ${ }^{11}$.

\section{MATERIALES Y MÉTODOS}

Diseño. El presente estudio siguió un enfoque cuantitativo, observacional, descriptivo, prospectivo, transversal y analítico, se empleó la lista de cotejo STROBE para la fabricación de un diseño epidemiológico de tipo transversal'12. Se ejecutó en el Instituto Nacional Materno Perinatal en la ciudad de Lima, Perú. Se inició la recolección de datos el 18 de enero del 2021 y finalizó el 23 de enero del 2021.

Población y Muestra. La población objetivo se compuso por un total de 1000 madres primíparas atendidas por teleconsulta en el INMPN en el año 2020. Para el cálculo de tamaño muestral se empleó el paquete estadístico EPIDAT versión 4.2, donde se tomó en cuenta un total poblacional de 1000 , intervalos de confianza del $95 \%$, una precisión del $5 \%$ y una frecuencia esperada de $23.8 \%$ para un nivel de conocimiento alto, reportada por Velasquez et $a l^{13}$, con lo que se obtuvo un tamaño muestral de 218 pacientes. Se adiciono un $10 \%$ al tamaño muestral para compensar aquellas perdidas que se pudieran presentar tomando en cuenta los criterios de selección establecidos para el presente estudio, con lo que se obtuvo un total de 240 gestantes primíparas atendidas por teleconsulta.

Se realizó un muestreo de tipo probabilísticoaleatorio simple sin reposición, empleando el paquete estadístico Epidat para la selección 240 individuos de estudio requeridos para la muestra. Como criterios de selección se incluyó a las madres primíparas que dieron su consentimiento informado para participar en la investigación y a las madres primíparas que cuenten con conexión a internet para poder usar la red social WhatsApp. Se excluyó del estudio a las madres que tienen habilidades distintas, que tienen enfermedades psiquiátricas, analfabetas, aquellas cuya lengua sea diferente al castellano y a las madres que no respondieron parte del cuestionario. Tomando en consideración estas características se excluyó un total de 14 gestantes del estudio, por lo que se trabajó con 226 pacientes.

Variables e instrumentos. El nivel de conocimiento sobre lactancia materna exclusiva se midió empleando un instrumento generado y validado por León et al, el cual posee 10 ítems, una validez de contenido por juicio de expertos y un grado de confiabilidad adecuado dado por el coeficiente alfa de Cronbach de $88 \%$. Este se categorizó como alto con un resultado mayor de 6 puntos, medio cuando con un resultado de 3 a 6 puntos y bajo con un resultado menor de 3 puntos. Se tomaron como variables modificadoras de la variable de interés al grado de instrucción, la gestante añosa, edad, el estado civil, antecedente de aborto recurrente y número de abortos. Para definir la variable madre añosa se tomó como punto de corte una edad mayor igual a 35 años, y para la variable aborto recurrente se consideró haber presentado 2 o más abortos.

La encuesta se aplicó de forma virtual para lo cual se contactó a las pacientes por medio de la aplicación WhatsApp; a través de esta se les envió un formulario de Google Forms, para su autollenado, para acceder a la encuesta, la paciente debía aceptar o declinar el consentimiento informado presentado en la primera parte del formulario. La información recolectada fue posteriormente enviada al programa Excel.

Análisis Estadístico. Se representó la distribución de frecuencias de cada una de las categorías de la variable nivel de conocimiento de lactancia materna exclusiva en una tabla de distribución de frecuencias y un gráfico de 
barras, se presentaron intervalos de confianza al 95\% para la prevalencia de nivel de conocimiento de lactancia materna alta. Las variables cualitativas gestante añosa, aborto recurrente, grado de instrucción y estado civil se representaron en una tabla de distribución de frecuencias.

Posteriormente, se reportó una tabla de contingencia la frecuencia absoluta y relativa de las variables gestante añosa, aborto recurrente grado de instrucción, estado civil por nivel de conocimiento de lactancia materna alto y no alto, a su vez se empleó la prueba estadística chi2 de Pearson cuando los valores esperados fueran menores a 5 , y en su defecto se trabajó con la prueba exacta de Fisher, para ambas pruebas se estableció un alfa critico de 0.05 para determinar asociación.

Se realizó un modelo de regresión de Poisson binario bivariado para las variables gestante añosa, aborto recurrente, grado de instrucción y estado civil, para obtener la razón de prevalencia y sus respectivos intervalos de confianza.

Con aquellas variables que demostraron una fuera de asociación significativa Se realizó un modelo de regresión de Poisson múltiple bivariado para obtener la razón de prevalencia ajustada y sus respectivos intervalos de confianza.

Aspectos Éticos. Todas las participantes del presente trabajo llenaron debidamente el consentimiento informado para el uso de los datos generados en la encuesta en el trabajo de investigación. Se contó con la aprobación de consejo de facultad de medicina de la universidad Ricardo palma, la aprobación de la unidad funcional de investigación del instituto materno perinatal. Se contó con la aprobación del comité de ética del instituto nacional materno perinatal Por tratarse de un estudio cuya unidad de análisis son seres humanos.

\section{RESULTADOS}

Se encontró una frecuencia relativa del $75,7 \%$ para el nivel alto de conocimiento en lactancia materna exclusiva (LME), con intervalo de confianza establecido al 95\% del $69.5-81.1 \%$.

El $62,4 \%$ de los participantes convivía con su pareja, un $13,3 \%$ de la muestra se conformo por gestantes con edad mayor o igual a 35 años, un $61,9 \%$ tenía un grado de instrucción superior y el $11,9 \%$ había tenido como antecedente 2 o más episodios de aborto (Tabla 1).

Las madres convivientes tuvieron una mayor frecuencia de nivel de conocimiento alto de LME $(62 \%)$ en comparación a las casadas y solteras. $66,7 \%$ de las madres con un nivel de conocimiento alto de LME tuvieron un nivel de instrucción superior. Se encontró una asociación estadísticamente significativa entre madre añosa y nivel de instrucción con nivel de conocimiento alto de LME, reflejándolo un valor $\mathrm{p}$ de 0.04 y 0.01 respectivamente (Tabla 2).

En el modelo de regresión bivariado de Poisson se encontró que las variables madre añosa (RP 1.23 IC95\% $1,06-1,42$ ) y grado de instrucción superior (RP 1,23 IC $95 \% 1,03-1,46)$ presentaban una fuerza y dirección de asociación estadísticamente significativa para la variable conocimiento de LME alto (valor p 0.01 y 0.00 respectivamente) (Tabla 3 ).

En el modelo de regresión múltiple de Poisson se encontró que las variables madre añosa (RPa 1.21 IC95\% $1,05-1,39)$ y grado de instrucción superior (RP 1,22 IC $95 \% 1,02-1,45)$ presentaban una fuerza y dirección de asociación estadísticamente significativa para la variable conocimiento de LME alto (valor p 0.006 y 0.022 respectivamente) (Tabla 3 ).

Tabla 1. Tabla de distribución de frecuencias. Análisis univariado

\begin{tabular}{lcc}
\hline \multicolumn{1}{c}{ Variables } & $\mathbf{n}$ & $\%$ \\
\hline Conocimiento de LME & 2 & 0,9 \\
$\quad$ Bajo & 53 & 23,5 \\
\hline Medio & 71 & 75,7 \\
$\quad$ Alto & & \\
Estado civil & 43 & 19,0 \\
$\quad$ soltera & 42 & 18,6 \\
\hline casada & 141 & 62,4 \\
$\quad$ conviviente & & \\
Madre añosa & 196 & 86,7 \\
$\quad$ No añosa & 30 & 13,3 \\
$\quad$ añosa & & \\
Grado de instrucción & 86 & 38,1 \\
$\quad$ No superior & 140 & 61,9 \\
$\quad$ superior & & \\
Aborto recurrente & 199 & 88,1 \\
$\quad$ No aborto recurrente & 27 & 11,9 \\
\hline Aborto recurrente & & \\
\hline
\end{tabular}

Tabla 2. Tablas de contingencia. Análisis bivariado

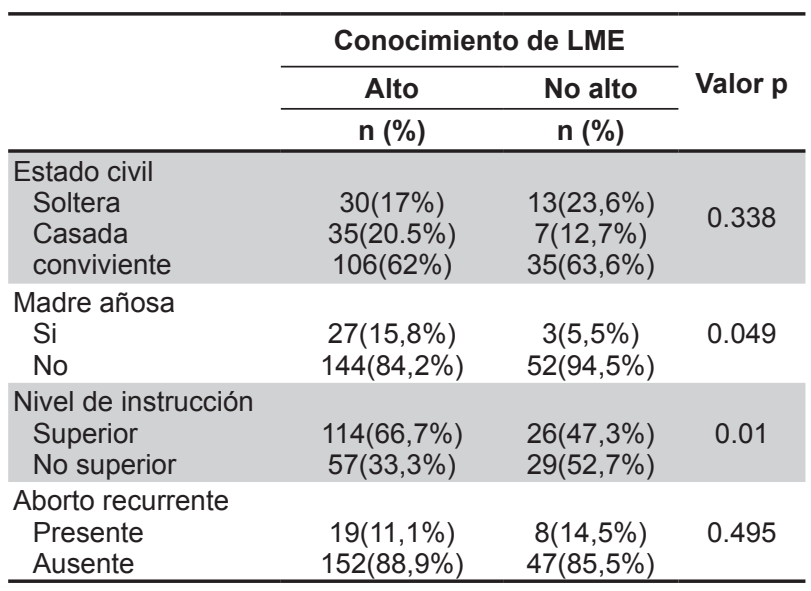


Tabla 3. Análisis de regresión de Poisson bivariado y múltiple.

\begin{tabular}{|c|c|c|c|c|c|c|}
\hline \multirow[b]{2}{*}{ Variable } & \multicolumn{3}{|c|}{ Regresión bivariado } & \multicolumn{3}{|c|}{ Regresión múltiple } \\
\hline & RP & IC $95 \%$ & Valor-P & $\mathrm{RPa}$ & IC $95 \%$ & Valor-P \\
\hline $\begin{array}{l}\text { Grado de } \\
\text { Instrucción } \\
\text { superior }\end{array}$ & 1.23 & $1.03-1.46$ & 0.01 & 1.22 & $1.02-1.45$ & 0.022 \\
\hline Madre añosa & 1.23 & $1.06-1.42$ & 0.00 & 1.21 & $1.05-1.39$ & 0.006 \\
\hline $\begin{array}{l}\text { Aborto } \\
\text { recurrente }\end{array}$ & 0.92 & $0.71-1.19$ & 0.53 & & & \\
\hline Estado civil & 1.19 & $0.94-1.52$ & 0.14 & & & \\
\hline
\end{tabular}

Abreviaturas: RP, Razón de prevalencia; IC, Intervalo de confianza; $R P a$, Razón de prevalencia ajustado

\section{DISCUSIÓN}

Nuestra investigación encontró que las madres presentaron un nivel de conocimiento de LME alto del $75.7 \%$, medio del $23,5 \%$ y bajo del $0,9 \%$. Estos resultados fueron diferentes a los encontrados previos a la pandemia como los de Tasayco et al(2017), donde se encuentra un nivel de conocimiento de LME alto del $25 \%$ en el primer nivel de atención ${ }^{13}$ y el de Álvarez et al (2019) que encontró un $11.3 \%$ también para nivel de conocimiento alto en nuestro instituto ${ }^{14}$. Si bien puede ser que las diferencias de los resultados estén relacionadas al nivel de atención de las gestantes, es muy probable que exista una fuerte asociación con el tipo de atención mixta que reciben las gestantes en nuestro hospital el cual consiste en una atención cercana vía teleconsultas alternadas con citas presenciales todo coordinado por un equipo de coordinadoras encargadas de gestionar todas sus atenciones. Ellas al estar cercanamente monitorizadas mediante Teleconsultas por un médico Gíneco-obstetra que la atiende durante toda su gestación, y en constante orientación por un equipo de obstetras y enfermeras capacitadas en lactancia materna que están a disposición diaria y a toda hora para absolver y redireccionar cualquier duda relacionada al embarazo, tienen a disposición una maquinaria de información validada para que su nivel de conocimiento en lactancia y cualquier otro aspecto del embarazo sea bastante adecuado.

Según la comprobación de hipótesis encontramos una asociación estadísticamente significativa entre nivel de conocimiento de LME y las variables grado de instrucción y madre añosa, lo cual es similar al resultado del estudio realizado por Muñoz et al (2017) en el que también encuentran que las variables edad materna y grado de instrucción asociados significativamente al nivel de conocimiento de lactancia materna exclusiva ${ }^{15}$. El estudio realizado por Reynoso (2018) sobre factores asociados al nivel de conocimientos sobre lactancia materna exclusiva en las puérperas atendidas en el hospital vitarte, enero 2018, concluye al igual que nosotros que no hay asociación entre estado civil, aborto recurrente y nivel de conocimiento sobre lactancia materna exclusiva ${ }^{16}$. Otro estudio determinó que los predictores de un menor nivel de conocimiento sobre LME fueron educación profesional, sobrepeso y vivir en un área rural, ${ }^{17}$ variables que no consideramos en nuestro estudio.

Un hallazgo particular realizado en Inglaterra encontró que las madres adolescentes, consideradas a las menores de 20 años, tenían pobre conocimiento sobre lactancia materna y además eran más frecuentemente solteras, tenían poco grado de educación, mayor desempleo, mayor frecuencia de hábito de fumar y menos contacto con alguna persona que haya amamantado previamente. Estos nos dan pie a nuevas investigación en adolescentes, las cuales tienen diferentes variables que aumentan su vulnerabilidad durante su gestación ${ }^{18}$.

Dentro las limitaciones del trabajo es importante mencionar que el presente estudio por ser unicéntrico, no incluyó dentro de su población pacientes de otras IPRESS tales como ESSALUD, establecimientos privados, de las fuerzas policiales, entre otras, esto afectaría la extrapolación de los datos a Lima metropolitana pues hay una marcada diferencia entre los estratos sociales de nuestra población objetivo respecto a las demás IPRESS. Por otro lado, se desarrolló en un establecimiento de salud nivel III-2, lo cual afecta la extrapolación de los resultados a la totalidad de las gestantes atendidas en la IPRESS MINSA, pues la mayoría de las gestantes tiene algún antecedente clínico que ha generado su atención en un Instituto materno y no en un establecimiento de menor nivel.

Recomendamos para futuros estudios plantear la hipótesis de cuánto se asocia la atención prenatal mixta con el nivel de conocimiento de LME comparando poblaciones que no haya recibido la orientación de Teleconsultas con las que sí, para reafirmar el hecho de que un control cercano "a la palma de la mano" es un buen camino para conseguir gestantes empoderadas y con un embarazo y puerperio sano. Finalmente concluimos que esta estrategia que fue forjada para conservar el control prenatal de la gestante de alto riesgo obstétrico durante la emergencia sanitaria COVID-19 tiene todo el potencial para estructurar y protocolizar en siguientes investigaciones todo tipo de orientaciones, así como evaluar diferentes resultados materno perinatales en esta particular población.

Declaración de conflictos de intereses. Los autores declaran no tener conflicto de intereses.

Agradecimientos. Agradecemos y rescatamos la loable función de las licenciadas Yoli Tolentino Huaman, Rossana Robalino Diaz, Encarnación Espinoza, Lucrecia Peltroche La Rosa, Maria Alva Diaz, Evelyn Valdez, Hilda Micalay, Sandra Zaraza, Dora Gamarra Avila, Rosario Zevallos Galarza, Sonia Chinga, Carmen del Carmen, 
Sonia Tapia, Marlene Huamani, Amparo Polanco y Analy Tinoco; y de las orientadoras Ninette Miriam Mendez Velarde y Rosmery Ortiz quienes con sensibilidad, empatía y compromiso brindan desde el primer contacto con las gestantes una atención de calidad en todo momento representando un pilar indispensable de este nuevo sistema de atención.

Contribuciones de autoría. El autor participó en la génesis de la idea, diseño de proyecto, recolección e interpretación de datos, análisis de resultados y preparación del manuscrito del presente trabajo de investigación.

Financiamiento. Autofinanciado.

\section{REFERENCIAS BIBLIOGRÁFICAS}

1. Salazar S, Chávez M, Delgado X, Eudis Rubio TP. Lactancia materna. Arch Venez Pueric Pediatría 2009;72(4):163-6.

2. Urquizo Aréstegui R. Lactancia materna exclusiva: ¿siempre? Rev Peru Ginecol Obstet 2014;60(2):171-6.

3. Ospina JM, Urrego ÁMJ, Betancourt EAV. La importancia de la lactancia en el desarrollo físico, psíquico y relacional del niño. Vínculo 2015;12(1):07-18

4. Lactancia materna: ventajas, técnica y problemas [Internet]. [citado $2021 \mathrm{abr}$ 2];Available from: https://www. pediatriaintegral.es/publicacion-2015-05/lactancia-maternaventajas-tecnica-y-problemas/

5. Tuñoque CV. Las primeras comidas del niño. 2007;8.

6. Los beneficios de la lactancia materna para la madre [Internet]. HealthyChildren.org [citado 2021 mar 4];Available from: https://www.healthychildren.org/Spanish/ages-stages/ baby/breastfeeding/Paginas/benefits-of-breastfeeding-formom.aspx

7. León-Cava N, Pan American Health Organization, LINKAGES Project, editores. Quantifying the benefits of breastfeeding: a summary of the evidence. Washington DC: Food and Nutrition Program, Pan American Health Organization: LINKAGES Project, Academy for Educational Development; 2002.

8. Quispe Ilanzo MP, Oyola García AE, Navarro Cancino M, Silva Mancilla JA. Características y creencias maternas asociadas al abandono de la lactancia materna exclusiva. Rev Cuba Salud Pública 2015;41(4):0-0.
9. Factores VasquezAtoche Maria.pdf [Internet]. [citado 2021 mar 4];Available from: http://repositorio.upch.edu.pe/ bitstream/handle/upch/3955/Factores VasquezAtoche Maria.pdf?sequence=1\&isAllowed=y

10. Brahm $P$, Valdés V. Beneficios de la lactancia materna y riesgos de no amamantar. Rev Chil Pediatría 2017;88(1):0714.

11. Tirano Bernate DC, Pinzón Espitia OL, González Rodríguez JL, Tirano Bernate DC, Pinzón Espitia OL, González Rodríguez JL. Factores de riesgo y barreras de implementación de la lactancia materna: revisión de literatura. Rev Esp Nutr Humana Dietética 2018;22(4):263-71.

12. STROBE_checklist_v4_combined.pdf [Internet]. [citado 2021 mar 30];Available from: https://www.strobe-statement.org/ fileadmin/Strobe/uploads/checklists/STROBE_checklist_v4_ combined.pdf

13. Tasayco DCT. Conocimiento y práctica de lactancia materna exclusiva en madres de niños menores de 6 meses, Puesto de Salud Magdalena Nueva, Chimbote, 2017. :117.

14. Solís-Rojas, M., Salazar-Salvatierra, E., \& Huamán-Lahura, R. del C. (2019). Factores asociados al inicio de la lactancia materna precoz. Revista Peruana De Investigación Materno Perinatal, 7(2), 31-36. https://doi.org/10.33421/inmp.2018115

15. Guerrero $\mathrm{MH}$. Factores asociados al conocimiento de lactancia materna en puérperas hospitalizadas en el servicio de gineco-obstetricia del hospital "San José" Callao-Lima, Julio-Setiembre 2017. :64.

16. Reynoso Sanchez, Antonella E. Factores asociados al nivel de conocimientos sobre lactancia materna exclusiva en las puérperas atendidas en el Hospital Vitarte, enero 2018. Univ Ricardo Palma [Internet] 2018 [citado 2021 abr 2];Available from: http://repositorio.urp.edu.pe/handle/URP/1293

17. Zielińska MA, Sobczak A, Hamułka J. Breastfeeding knowledge and exclusive breastfeeding of infants in first six months of life. Rocz Panstw Zakl Hig 2017;68(1):51-9.

18. Dewan N, Wood L, Maxwell S, Cooper C, Brabin B. Breastfeeding knowledge and attitudes of teenage mothers in Liverpool. J Hum Nutr Diet Off J Br Diet Assoc 2002;15(1):33-7.

\section{Correspondencia:}

Pablo Gerardo Yutaka Vizquerra Guevara.

Dirección: Marítima baja Mz. N Lt. 149, puerto Chancay

E-mail: pablo.vizquerra@urp.edu.pe

Teléfono: 983716442 